\title{
The D234 action for light quarks
}

\author{
M. Alfordt, T. Klassent, and P. Lepage \\ Newman Laboratory of Nuclear Studies \\ Cornell University \\ Ithaca, NY 14853 \\ USA
}

We investigate a new light fermion action (the "D234" action), which is accurate up to $\mathcal{O}\left(a^{3}\right)$ and tadpoleimproved $\mathcal{O}\left(a \alpha_{s}\right)$ errors. Using D234 with Symanzik- and tadpole-improved glue we find evidence that continuum results for the quenched hadron spectrum (pion, rho and nucleon) can be obtained on coarse lattices.

\section{INTRODUCTION}

It has recently become clear that improved actions [ 1, 2] are a powerful and practical tool for obtaining continuum results from the lattice. This has been demonstrated for the $\Upsilon$ with the non-relativistic QCD action [3], and also for the static quark potential and charmonium with improved glue [ 1 . In the latter case, continuum results were obtained from lattices as coarse as $a=0.4 \mathrm{fm}$.

At tree level, improved glue has $\mathcal{O}\left(a^{4}\right)$ errors, much smaller than the $\mathcal{O}\left(a^{2}\right)$ errors of the Sheikholeslami-Wohlert (SW) action [ [5] for fermions, which is the most improved light quark action used in simulations up to now (see other contributions at this conference). In order to bring the quark action up to the same level of accuracy as the glue, we have started a program of investigating improved light fermion actions. We here report results for the "D234" action in the quenched approximation. The D234 action uses a cubic derivative term to improve the naive lattice Dirac operator. It also contains second and fourth order derivative terms (as well as the $\sigma \cdot F$ "clover" term) with fine tuned coefficients to eliminate the "spatial doublers" at large momentum and minimize the number of "time doublers" at low momentum without introducing errors larger

\footnotetext{
*Address after Sept 1995: School of Natural Sciences, Institute for Advanced Study, Princeton, NJ 08540

${ }^{\dagger}$ Address after Sept 1995: SCRI, Florida State University, Tallahassee, FL 32306
}

than $\mathcal{O}\left(a^{3}\right)$.

As with other improved actions, we find that tadpole improvement [ 6] of the coefficients is essential in obtaining accurate results with the D234 action. At tree level it can be implemented by simply replacing each gauge link $U$ in the action by $\tilde{U}=U / u_{0}$, where $\left(u_{0}\right)^{4}$ is the expectation value of the plaquette.

\section{D234 ACTION}

The D234 fermionic operator used in the simulations described here is:

$$
\begin{aligned}
& m_{0}+\sum_{\mu}\left(\gamma_{\mu} \Delta_{\mu}^{(1)}-b a^{2} \gamma_{\mu} \Delta_{\mu}^{(1)} \Delta_{\mu}^{(2)}\right. \\
& \left.-\frac{r a}{2}\left(\Delta_{\mu}^{(2)}+\frac{1}{2} \sum_{\nu} \sigma_{\mu \nu} F_{\mu \nu}\right)+c a^{3} \Delta_{\mu}^{(2)} \Delta_{\mu}^{(2)}\right)
\end{aligned}
$$

where $b=1 / 6, r=2 / 3, c=1 / 12$,

$$
\begin{aligned}
\Delta_{\mu}^{(1)} \psi(x)= & \frac{1}{2 a}\left(\tilde{U}_{\mu}(x) \psi(x+\mu)\right. \\
& \left.-\tilde{U}_{-\mu}(x) \psi(x-\mu)\right), \\
\Delta_{\mu}^{(2)} \psi(x)= & \frac{1}{a^{2}}\left(\tilde{U}_{\mu}(x) \psi(x+\mu)\right. \\
& \left.+\tilde{U}_{-\mu}(x) \psi(x-\mu)-2 \psi(x)\right),
\end{aligned}
$$

and for $F_{\mu \nu}$ we use the clover representation [5].

Classically the D234 action has $\mathcal{O}\left(a^{3}\right)$ errors. One can show this by using a change of variable to relate it to $\gamma_{\mu} \Delta_{\mu}^{(1)}\left(1-\frac{a^{2}}{6} \Delta_{\mu}^{(2)}\right)$, which differs 
at $\mathcal{O}\left(a^{4}\right)$ from the continuum Dirac operator. For the rest of this paper we ignore the change of variable since it does not affect spectral quantities. At the 1-loop level there are additional $\mathcal{O}\left(a g^{2}\right)$ errors. The coefficients $r$ and $c$ are chosen so that there is only one low momentum doubler in the free dispersion relation, as can be seen in fig. 1. For the glue we used the Symanzik- and tadpoleimproved action described in [2, 国, which has $O\left(a^{4}\right)$ and $O\left(a^{2} g^{2}\right)$ errors.

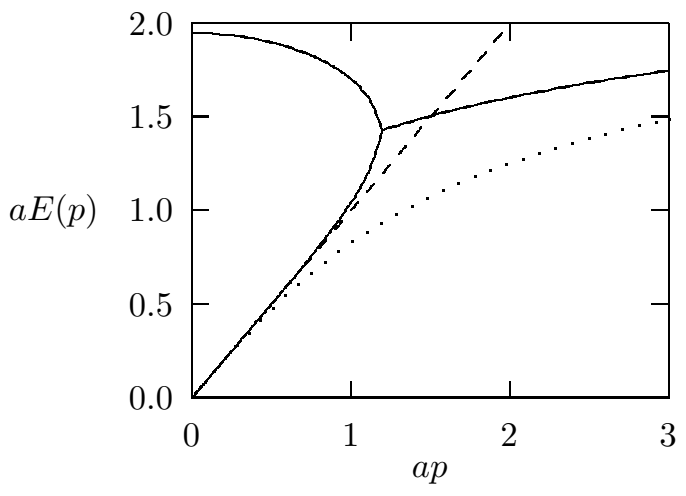

Figure 1. Massless dispersion relations for free D234 (solid lines), Wilson or SW (dotted line) and continuum (dashed line) fermions. Momentum $p$ is along the $(1,1,0)$ direction. Beyond the D234 branch point, the real part of the two conjugate roots is shown.

\section{PROCEDURE}

The results reported here were obtained using code written in $\mathrm{C}++$ (with matrix multiplications in FORTRAN for added speed), and run on Cornell's SP-2. Gluon configurations were generated by the Metropolis algorithm, with 10 hits per update and $20-30$ updates between configurations, which suffices to decorrelate them on the volumes we used. The quark propagator in each gauge background was found using the BiCGStab2 algorithm, supplemented by a simple type of preconditioning.
We performed simulations at three couplings, $\beta \equiv \beta_{\mathrm{pl}}=6.8,7.1,7.4$, corresponding to lattice spacings $a=0.40,0.33,0.24 \mathrm{fm}$, if one uses charmonium to set the scale [ []. We have results for seven quark masses at $\beta=6.8$ and 7.1 , for four at $\beta=7.4$, with $m_{\rho} / m_{\pi}$ ratios in the range between 1.2 to 2.0. For the smaller ratios the spatial size of our lattices is about $2.0 \mathrm{fm}$, increasing to $2.6-$ $3.2 \mathrm{fm}$ for larger ratios, except for $\beta=7.4$, where at present our largest lattices are $2.2 \mathrm{fm}$. In lattice units our spatial volumes are in the range $5^{3}$ to $9^{3}$. The time extent of our lattices is always as least twice the spatial one. Since the cost of generating gluon configurations is negligible compared to that of the inversion, we did not save configurations but generated independent ones for each quark mass. We typically produced between 200 and 600 propagators per quark mass, the smaller numbers corresponding to $\beta=7.4$.

We calculated the time slice propagators for the relativistic pion, rho and nucleon operators, as well as for the non-relativistic nucleon, and the $J_{z}=1 / 2$ and $3 / 2$ delta operators. Furthermore, we obtained the pion and rho with various momenta. In all cases we suitably averaged over spin so that (in the infinite statistics limit) the time slice propagators for large times $t$ are $\propto \cosh m\left(\frac{T}{2}-t\right)$, where $\mathrm{T}$ is the time extension of the lattice.

We used a covariant form of gaussian smearing for the quark fields at the source and sink, employing the operator $\left(1+\epsilon \sum_{i} \Delta_{i}^{(2)}\right)^{N}$. On our coarse lattices it seems that for a given $\beta$ one can use the same, near-optimal (in terms of cost/error) value of $\epsilon N$ for all quark masses and hadrons. Initially we used (correlated) $2 \times 2$ matrix fits of all four combinations of sink and source local and smeared propagators (LL, SL, LS, SS) to ground and excited state cosh's. After determining the values of $\epsilon N$ where the SS propagator has only a very small excited state contribution, we found it more cost efficient to generate only the source smeared LS and SS propagators and fit them to a single cosh, either separately or in a combined fit. We used a reliable, if perhaps somewhat conservative method to combine effective mass estimates from fits with different $t_{\min }$, 
instead of picking the fit with the best "goodness" by some criterion. This is relevant in cases where fits from different $t_{\min }$ appear to be nearly equally "good".

\section{RESULTS}

We first investigated finite volume effects. We find that for $m_{\rho} / m_{\pi} \approx 2$, infinite volume results are reached within our statistical errors for the pion and rho at spatial sizes of around $2.0 \mathrm{fm}$, for the nucleon around $2.5 \mathrm{fm}$. For smaller $m_{\rho} / m_{\pi}$ ratios, somewhat smaller volumes suffice. All our results presented below should therefore have no significant finite volume errors, except perhaps the nucleon at $\beta=7.4$ (cf. below). The delta might require even bigger volumes than the nucleon, and we will not present results for it, except to remark that the good agreement we find between the masses of the $J_{z}=1 / 2$ and $3 / 2$ delta states, which are as large as $a m_{\Delta} \approx 3$, provide good evidence for the rotational invariance of the D234 action on coarse lattices.

Further such evidence is furnished by the pion and rho states with momenta. Defining a "speed of light" $c$ via

$c^{2} p^{2}=E(p)^{2}-E(0)^{2}$,

we compare in table 11 results from the D234 and SW actions. We see that rotational invariance, i.e. $c^{2} \approx 1$, is restored already on the coarsest lattice for D234, in contrast to SW. This conclusion is even more striking for the higher momenta we investigated. We have also checked that $c$ deteriorates dramatically without tadpole improvement in the D234 action, even though it is still better than for SW with tadpole improvement (with improved glue in both cases).

We now describe our results for the hadron masses. The data are shown in table 2. For $m_{\rho}$ we can obtain perfect linear fits to $V_{0}+V_{2} m_{\pi}^{2}$ up to the largest masses considered, as shown in table 3 , and in fig. 2 for $\beta=7.1$. Lacock and Michael [7] proposed to use the dimensionless parameter $J=m_{V} d m_{V} / d m_{P}^{2}$ at $m_{V} / m_{P}=m_{K^{\star}} / m_{K}=1.8$ to characterize the vector versus pseudo-scalar mass relation and compare lattice studies to nature. They estimate $J=0.48(2)$ for the real world
Table 1

"Speed of light" squared $\left(c^{2}\right.$ in eq. 4) for mesons with momentum $p=2 \pi / a L, a L \approx 2.0 \mathrm{fm}$ at $m_{\pi} / m_{\rho} \approx 0.70$.

\begin{tabular}{lllll}
\hline \multicolumn{3}{l}{ D234 Action } & \multicolumn{2}{l}{ SW Action } \\
$\beta$ & $\pi$ & $\rho$ & $\pi$ & $\rho$ \\
\hline 6.8 & $0.95(2)$ & $0.93(3)$ & $0.63(2)$ & $0.48(3)$ \\
7.1 & $0.94(3)$ & $0.96(5)$ & $0.74(3)$ & $0.55(4)$ \\
7.4 & $0.99(4)$ & $1.00(6)$ & - & - \\
\hline
\end{tabular}

- note that $J=0.5$ if $m_{V}^{2}-m_{P}^{2}$ were exactly constant - and $J=0.37(2)(4)$ as the "world average" from quenched simulations. Our estimate of $J$ from chiral fits is shown in table 5 . Other estimates in these proceedings are also in excellent agreement with ours, but do not scale on similarly coarse lattices.

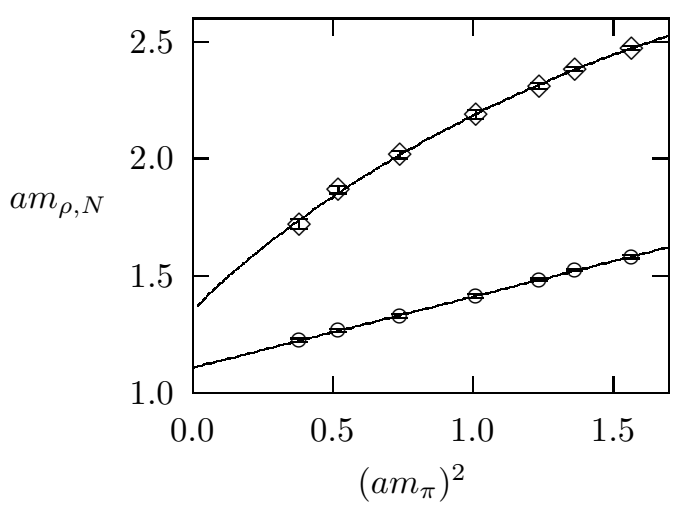

Figure 2. Masses of the rho (circles) and nucleon (diamonds) and their chiral fits at $\beta=7.1$.

We now turn to the nucleon. The masses extracted from the relativistic and non-relativistic operators are found to agree well within statistical errors. To obtain good chiral fits it is crucial, in general, to include a cubic term, i.e. fit $m_{N}$ to $N_{0}+N_{2} m_{\pi}^{2}+N_{3} m_{\pi}^{3}$, cf. fig 2. Our results are shown in table 4 . For $\beta=7.4$ we can not completely exclude finite volume errors in the chirally extrapolated $m_{N}$, but they are almost certainly smaller than the large statistical errors we have 
Table 2

Light hadron masses from the D234 action

\begin{tabular}{cclll}
\hline$\beta$ & $a m_{0}$ & $a m_{\pi}$ & $a m_{\rho}$ & $a m_{N}$ \\
\hline 6.8 & -0.15 & $0.700(4)$ & $1.375(10)$ & $2.047(24)$ \\
& -0.08 & $0.911(4)$ & $1.466(8)$ & $2.270(17)$ \\
& -0.05 & $0.980(3)$ & $1.497(6)$ & $2.331(16)$ \\
& 0 & $1.092(3)$ & $1.566(6)$ & $2.464(15)$ \\
& 0.05 & $1.206(3)$ & $1.638(5)$ & $2.598(12)$ \\
& 0.1 & $1.298(2)$ & $1.698(5)$ & $2.695(10)$ \\
& 0.2 & $1.480(3)$ & $1.838(5)$ & $2.951(14)$ \\
\hline 7.1 & -0.14 & $0.616(4)$ & $1.226(8)$ & $1.720(21)$ \\
& -0.11 & $0.721(3)$ & $1.266(5)$ & $1.868(17)$ \\
& -0.06 & $0.861(3)$ & $1.327(7)$ & $2.018(16)$ \\
& 0 & $1.005(3)$ & $1.413(7)$ & $2.189(19)$ \\
& 0.05 & $1.112(2)$ & $1.483(5)$ & $2.311(15)$ \\
& 0.08 & $1.168(2)$ & $1.524(4)$ & $2.384(10)$ \\
& 0.12 & $1.251(2)$ & $1.581(6)$ & $2.474(10)$ \\
\hline 7.4 & -0.05 & $0.662(4)$ & $1.055(9)$ & $1.560(15)$ \\
& 0 & $0.806(4)$ & $1.147(7)$ & $1.739(10)$ \\
& 0.05 & $0.921(5)$ & $1.224(9)$ & $1.869(14)$ \\
& 0.1 & $1.031(3)$ & $1.304(4)$ & $2.010(9)$ \\
\hline
\end{tabular}

Table 3

Chiral fit parameters (in lattice units) of the rho mass

\begin{tabular}{lllll}
\hline$\beta$ & $V_{0}$ & $V_{2}$ & $N_{\mathrm{DF}}$ & $Q$ \\
\hline 6.8 & $1.238(7)$ & $0.274(5)$ & 5 & 0.98 \\
7.1 & $1.108(6)$ & $0.303(5)$ & 5 & 0.94 \\
7.4 & $0.889(13)$ & $0.390(14)$ & 2 & 0.67 \\
\hline
\end{tabular}

in this case. In table 5 we show our estimates of $m_{N} / m_{\rho}(=1.22$ in nature) in the chiral limit.

So far the results described for the D234 action have been spectacular. There is however one problem, which is revealed in table 5: the scale determined from the chirally extrapolated $\rho$ mass (set to $770 \mathrm{MeV}$ ) does not agree with that from the charmonium $1 \mathrm{~S}-1 \mathrm{P}$ splitting $\left(a_{\psi}\right)$, which is known to scale quite well [ []. In view of the excellent properties of $c^{2}$ and $J$ we interpret this as indicating a small additive shift of all masses (vanishing, of course, as $a \rightarrow 0$ ) that depends, it seems, only rather weakly on the momentum or type of the particle, at least for mesons. We are investigating whether the doublers are responsi-
Table 4

Chiral fit parameters (in lattice units) of the nucleon mass

\begin{tabular}{llllll}
\hline$\beta$ & $N_{0}$ & $N_{2}$ & $N_{3}$ & $N_{\text {DF }}$ & $Q$ \\
\hline 6.8 & $1.66(8)$ & $0.99(21)$ & $-0.29(13)$ & 3 & 0.91 \\
7.1 & $1.35(5)$ & $1.30(18)$ & $-0.47(12)$ & 4 & 0.80 \\
7.4 & $1.09(11)$ & $1.44(46)$ & $-0.56(35)$ & 1 & 0.55 \\
\hline
\end{tabular}

Table 5

Selected quantities relevant for scaling. $m_{N} / m_{\rho}$ is extrapolated to zero pion mass, $a_{\psi}$ is from [ [ [].

\begin{tabular}{lllll}
\hline$\beta$ & $J$ & $m_{N} / m_{\rho}$ & $a_{\psi}^{-1}(\mathrm{MeV})$ & $a_{\psi} / a_{\rho}$ \\
\hline 6.8 & $0.384(8)$ & $1.34(6)$ & $497(4)$ & $1.251(12)$ \\
7.1 & $0.381(8)$ & $1.22(5)$ & $606(5)$ & $1.147(11)$ \\
7.4 & $0.395(18)$ & $1.23(12)$ & $821(10)$ & $1.055(20)$ \\
\hline
\end{tabular}

ble for this shift. There are various strategies to "push away" the doublers, and we are hopeful that a completely satisfactory fermionic action for coarse lattices can be found.

Acknowledgements. This research was conducted using the resources of the Cornell Theory Center, which receives major funding from the NSF and New York State, with additional support from ARPA, the National Center for Research Resources at the NIH, IBM and other members of the center's Corporate Research Institute.

\section{REFERENCES}

[1] K. Symanzik, Nucl. Phys. B226 (1983) 187.

[2] M. Lüscher and P. Weisz, Comm. Math. Phys. 97 (1985) 59; Phys. Lett. 158B (1985) 250.

[3] C. Davies et al, to be published in Phys. Rev. D (1995); Phys. Lett. B345 (1995) 42.

[4] M. Alford, W. Dimm, G. P. Lepage, G. Hockney, and P. B. Mackenzie, Nucl. Phys. B42 (Proc. Suppl.) (1995) 787 and hep-lat/9507010.

[5] B. Sheikholeslami and R. Wohlert, Nucl. Phys. B259 (1985) 572.

[6] G. P. Lepage and P. B. Mackenzie, Phys. Rev. D48 (1993) 2250.

[7] P. Lacock and C. Michael, hep-lat/9506009. 